\title{
Overexpression of ID1 reverses the repression of human dental pulp stem cells differentiation induced by TWIST1 silencing
}

\author{
Izabela Maciejewska1 ${ }^{1}$, Monika Sakowicz-Burkiewicz² ${ }^{2 凶}$, Marta Krzeminska ${ }^{1}$ and Tadeusz \\ Pawelczyk ${ }^{2}$ \\ Department of Dental Prosthodontics, Medical University of Gdansk, Gdańsk, Poland; Department of Molecular Medicine, Medical University of
} Gdansk, Gdańsk, Poland

\begin{abstract}
Multiple studies showed that the cessation of TWIST1 expression is the prerequisite for osteoblasts' maturation. However, recent reports revealed that the function of TWIST1 is different in the dental pulp stem cells

(DPSCs), where a high level of TWIST1 expression promoted DPSCs' differentiation. The aim of the study was to investigate the impact of TWIST1 and ID1 on the differentiation process in the human DPSCs. Methods: TWIST1 and ID1 expression in the DSPCs was modulated by lentivirus transduction. Genes expression was assessed with qRT-PCR. The proteins level was evaluated by Western blot. The DPSCs differentiation was assessed with the proliferation, alkaline phosphatase (ALP) activity, and calcium concentration assays. Results: TWIST1 silencing suppressed the expression of ID1 and both the early and late markers of odontoblasts' differentiation detected at the transcript and protein level. The forced overexpression of ID1 increased the expression of the late markers of odontoblasts differentiation but diminished the expression of the early markers. DPCSs with the silenced TWIST1 and subsequent ID1 overexpression displayed an increase in the expression of the late markers of odontoblasts differentiation. Cells with silenced TWIST1 and overexpressing ID1 had increased activity of $A L P$, higher calcium concentration and decreased proliferation rate. The high level of ID1 expression might be a critical factor stimulating DPSCs differentiation and it might compensate the repressed differentiation of DPSCs caused by TWIST1 silencing. Conclusion: The mutual correlation between the expression level of TWIST1 and ID1 might be a critical factor driving the process of the human odontoblasts' differentiation.
\end{abstract}

Key words: dental pulp stem cells, TWIST1, ID1, odontoblast differentiation, DSPP, DMP1

Received: 03 February, 2017; revised: 07 September, 2017; accepted: 02 October, 2017; available on-line: 20 November, 2017

e-mail: ssak@gumed.edu.pl

Abbreviations: DPSC, dental pulp stem cell; TWST1, twist family BHLH transcription factor 1; ID1, inhibitor of DNA binding 1

\section{INTRODUCTION}

The expression of TWIST1 undergoes precise spatiotemporal regulation, which mediates the development of tissues and organs of the mesenchymal origin. The phenotype assigned to TWIST1 results from TWIST1 binding to its dimerization partners and might occur as a transcriptional repression and/or activation (Connerney et al., 2006; Connerney et al., 2008; Thisse et al., 1991; Zhang et al., 2008). TWIST1 expression pattern was thoroughly examined in bone, where the cessation of TWIST1 expression was claimed as the critical signal for osteoblasts differentiation (Bialek et al., 2004). It was shown that TWIST1 homodimers enhanced bone formation and mineralization while TWIST1 heterodimers with E12 protein repressed osteoblasts differentiation and subsequent bone mineralization (Connerney et al., 2006). This mechanism of TWIST1 dimerization was discovered during Drosophila mesoderm development (Castanon et al., 2001) but seems more complicated in mammals (Connerney et al., 2006). The complexity of TWIST1/ E12 (T/E) heterodimer function in mice was reported by Laursen and coworkers who showed that murine $\mathrm{T} / \mathrm{E}$ heterodimer drove the reporter construct coming from Drosophila, which originally was driven by TWIST1 homodimer (Laursen et al., 2007). Multiple studies on TWIST1 function showed that the cessation of TWIST1 expression is the prerequisite for the final osteoblasts maturation and subsequent bone mineralization (Rice et al., 2000; Rice et al., 2003; Rice et al., 2005), but the recent reports suggest that the high level of both TWIST1 and ID1 expression works differently in the dental pulp stem cells (DPSCs), where the overexpression of both genes pushed DPSCs into the final differentiation into odontoblasts-like cells (Li et al., 2011; Zhang et al., 2012). The in vivo study confirmed this observation proving that the stable expression of TWIST1 is critical for the dental organ development including odontoblasts differentiation and dental crown development (Meng et al., 2015).

Thus, considering that TWIST1 and ID1 are acting in concert to modulate the differentiation process of cells originating from mesenchyme and observing that these two genes might act in a cell specific manner we examined if the forced overexpression of ID1 in the DPSCs might rescue the repression of their differentiation caused by TWIST1 silencing.

\section{MATERIAL AND METHODS}

Patients and samples. Teeth for isolation of dental pulp stem cells (DPSCs) were obtained from 8 patients (13-22 years old) undergoing routine extractions in the Department of Oral Surgery, Medical University of Gdansk. The Institutional Review Board at the Medical University of Gdansk approved all procedures (NKEBN/427/2009-2010) and written consent was obtained from all patients.

Cells isolation and culture. DPSCs were isolated from the interrupted wisdom teeth as described previously (Gronthos et al., 2000). Obtained cells were cultured at density of $1 \times 10^{3} / \mathrm{cm}^{2}$ in DMEM supplemented 
with 15\% FBS, 2\% L-glutamine, $100 \mathrm{U} / \mathrm{mL}$ penicillin and $100 \mu \mathrm{g} / \mathrm{mL}$ streptomycin under standard conditions. The culture medium was replaced twice a week and cells were passaged at $70 \%$ confluence.

Cells selection. DPSCs with the phenotype STRO-1/+/; CD146/+/; CD34/-/; CD45/-/ were obtained by the positive selection with anti-STRO1 and anti-CD146 antibodies followed by a negative selection with mouse anti-human CD34 and CD45 antibodies employing the magnetic nanoparticles (MagCellect ${ }^{\mathrm{TM}}$ ) coated with goat anti-mouse $\operatorname{IgG}$ ferrofluid accordingly with the manufacturer protocol (all antibodies and MagCellect ${ }^{\mathrm{TM}}$ were from R\&D Systems, Minneapolis, MN, USA). The number of viable cells was determined by a hemocytometer-based Trypan Blue dye exclusion method. Cells were passaged at $80 \%$ confluence (usually the number of passages ranged from 3 to 4 ).

Lentivirus transduction driven TWIST1 knockdown. The TWIST1 silencing constructs: Twist-siRNA7 (\#8457), Twist-siRNA3 (\#1784) and Twist-siRNA5 (\#8456) by Bob Weinberg were from Addgene (Cambridge, MA, USA). The empty 3.7 pLL vector was used as a negative control. The culture media were used for DPSCs transduction. The packaging cells (HEK 293T) were co-transfected with either mix of all three constructs in the ratio 1:1:1 or empty 3.7 pLL vector, an envelope plasmid pMD2.G and a packaging plasmid psPAX2 using X-treme Gene 9 (Roche, Germany) according to the manufacturer's protocol. The culture medium was used as the resource of lentiviral particles for DPSCs transduction. The efficacy of DPSCs transduction was assessed by the observation of a GFP expression using a fluorescent microscope (Leica, Wetzlar, Germany). The DPSCs were cultured with the infectious medium until at least $80 \%$ of the population showed the presence of GFP and thereafter used for the further analysis. The silencing of TWIST1 expression was confirmed with the qPCR analysis.

Lentivirus transduction driven IDI overexpression. The human ID1 cDNA (Gen Bank, Accession No. X77956) was obtained by PCR and cloned into the pWPI-GFP lentiviral vector as described previously (Maciejewska et al., 2014). The empty pWPI-GFP vector was used as a negative control. The pWPI-GFP-ID1 lentiviral vector, together with pMD2.G plasmid and a packaging plasmid psPAX2, or the empty pWPI-GFP vector was used to transfect the HEK 293T cells. The medium with virus particles was used to transduce the wild DPSCs and DPSCs with silenced TWIST1 expression (siDPSCs). The everyday exposition of siDPSCs to the viral particles driving ID1 overexpression proceeded for a week, and the level of the ID1 overexpression was confirmed with the qRT-PCR.

Cultures of transduced cells. Transduced DPS cells were cultured in DMEM supplemented with 10\% FBS, $2 \%$ L-glutamine, $100 \mathrm{U} / \mathrm{mL}$ penicillin and $100 \mu \mathrm{g} / \mathrm{mL}$ streptomycin under standard conditions. For experiments DPSCs at 5 or 6 passage were plated in $100 \mathrm{~mm}$ dishes at a density of $2 \times 10^{4}$ cells and were cultured for 14 days with medium exchange every 3-4 days. The representative photographs of cultured cells are presented in Fig. 1.

Quantitative real-time PCR. Total RNA was isolated from DPSCs using STAT-60 (AMS Biotechnology, Abingdon, UK) according to the manufacturer's protocol. The levels of genes expression in the DPSCs were assessed with Quantitative PCR using a QuantiTect $^{\circledR}$ SYBR Green PCR kit (Qiagen, Germany). The primer sequences used are shown in Table 1S (at www. actabp.pl). Transcript levels were normalized to that of
$G A P D H$ using the $2^{\Delta \mathrm{Ct}}$ method. All reactions were performed in triplicate and validated by the presence of a single pick in the melting curve.

Western blot. Immunoblotting was performed on cell extract (Sakowicz-Burkiewicz et al., 2013) subjected to $4-20 \%$ resolving SDS-PAGE gel electrophoresis. The proteins separated were electrophoretically transferred onto PVDF membranes. The membranes were blocked with 3\% bovine serum albumin, incubated overnight with an appropriate primary antibody (Table $2 \mathrm{~S}$ at www. actabp.pl), followed by washing and incubation with a secondary antibody. $\beta$-actin was used as an internal reference protein.

Proliferation analysis. After 13 days of the cell culture DPSCs were plated at a density of $1 \times 10^{3}$ cells per well in 96 wells plate for 24 hours. Then, cells proliferation was measured with a Quick Cell Proliferation Assay Kit (Gentaur, Sopot, Poland) according to the manufacturer's instruction. The experiment was done in triplicate and repeated 5 times.

Alkaline phosphatase activity analysis. The activity of alkaline phosphatase (ALP) was determined with the QuantiChrom ${ }^{\text {TM }}$ Alkaline Phosphatase Assay Kit (Gentaur, Sopot, Poland) according to the manufacturer's recommendation. The absorbance (405 nm) was measured right after mixing and 5, 10 and 15 minutes thereafter using the plate reader Victor 3 (Perkin Elmer, Waltham, MA, USA). The ALP activity was calculated according to an equation given by the manufacturer. The experiment was done in triplicate and repeated 3 times.

Calcium concentration analysis. For the analysis of intracellular calcium concentration the cell extract
A

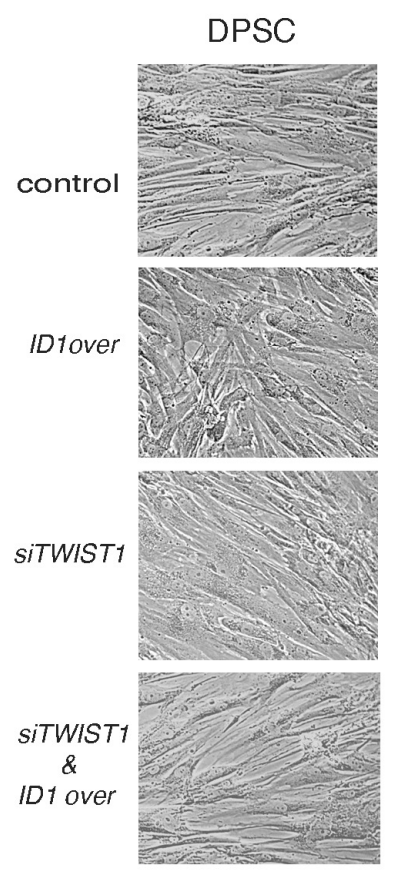

B

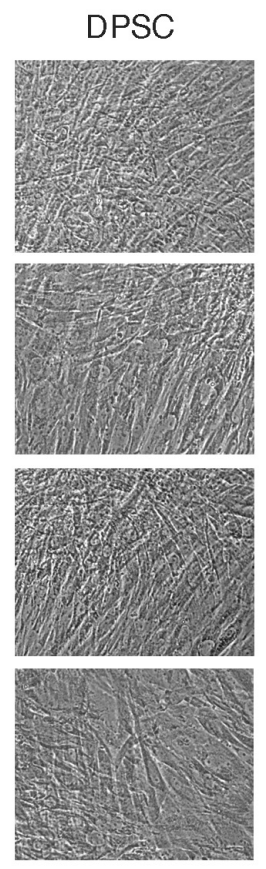

Figure 1. Light microscopy images of transduced DPSCs in culture.

The cells were transduced with an empty $3.7 \mathrm{pLL}$ vector (control), with pWPI-GFP-ID1 (ID1over), with 3.7 pLL vector carrying the TWIST1 SiRNAs (siTWIST1), with $3.7 \mathrm{pLL}$ vector carrying the TWIST1 siRNAs and subsequently with pWPI-GFP-ID1 vector (siTWIST1\&ID1over). (A) Representative microphotographs of 7-day-old cultures of transduced DPSC (20x magnification), (B) Representative microphotographs of 14-day-old cultures of transduced DPSC (10x magnification). 
was used. The procedure was performed using QuantiChrom $^{\mathrm{TM}}$ Calcium Assay Kit (Gentaur, Sopot, Poland) according to the manufacturer's recommendation. Calcium concentration was calculated according to the slope driven from standards included in the kit. The experiment was performed in triplicate and repeated 3 times.

Statistical analysis. Values are reported as means \pm S.D. Differences between groups were assessed using a Student's $t$-test. $P<0.05$ was considered as significant.

\section{RESULTS}

\section{ID1 overexpression compensates TWIST1 silencing} related restriction in the expression of differentiationrelated genes in DPSCs

qRT-PCR analysis showed that transduction of DPSCs with the equal mix of the three TWIST1-siRNA $(\# 3,5,7)$ lentiviral vectors resulted in the stable decrease of the TWIST1 expression down to $15 \%$, which last throughout the entire period of experiment regardless the subsequent modifications in the expression of ID1. We also observed that the transduction of DPSCs with the pWPI-GFP-ID1 resulted in the $\sim 5$-fold increase in the expression of ID1 regardless of TWIST1 expression status. This was visible both at the mRNA and protein level (Fig. 2A, 2B).

Silencing of TWIST1 expression resulted in the dramatic suppression of ID1 expression and expression of genes encoding both the early and late markers of odontoblasts differentiation i.e. ALP (alkaline phosphatase), DSPP (dentin sialophosphoprotein), DMP1 (dentin matrix acidic phosphoprotein protein 1), BSP (bone sialoprotein), and OSF2 (periostin). Concurrently, the mild decrease of the expression of $O N$ (osteonectin) and TSP1 (thrombospondin 1) was detected (Fig. 2A). The increase was observed only in the expression of $O C N$ (osteocalcin). The analogous observations were made on the basis of the Western Blot analysis (Fig 2B). Conversely, the forced overexpression of ID1 increased the subsequent expression of the late markers of odontoblasts differentiation i.e. DSPP, DMP1, BSP, ON, TSP1 at the transcript and protein level but diminished the expression of early markers OCN, ALP and OSF2 (Fig. 2A, 2B). Interestingly, in the DPSCs with the silenced TWIST1 and subsequent ID1 overexpression we observed an increase in the transcript level of BSP, ON, and DSPP. The changes in mRNAs correlated with the proteins levels (Fig. 2A, 2B).

\section{ID1 cooperate with TWIST1 in DPSCs differentiation into the odontoblast-like cells}

To supplement our observation coming from the qRT-PCR and WB analyses we measured the proliferation potential of DPSCs. The proliferation test showed that DPSCs with silenced TWIST1 and overexpressed ID1 proliferated significantly slower compared to the other cells (Fig. 3A). Measurements of ALP activity showed that cells overexpressing ID1 displayed the highest ALP activity regardless of the TWIST1 expression status. The lowest activity of ALP was detected in the DPSCs with silenced TWIST1 (Fig. 3B). Similarly, tests for calcium concentration showed that the DPSCs with forced overexpression of ID1 had the highest and the DPSCs with silenced TWIST1 the lowest calcium concentration. We noted significant difference in the level of calcium in DPSCs with silenced TWIST1 and the
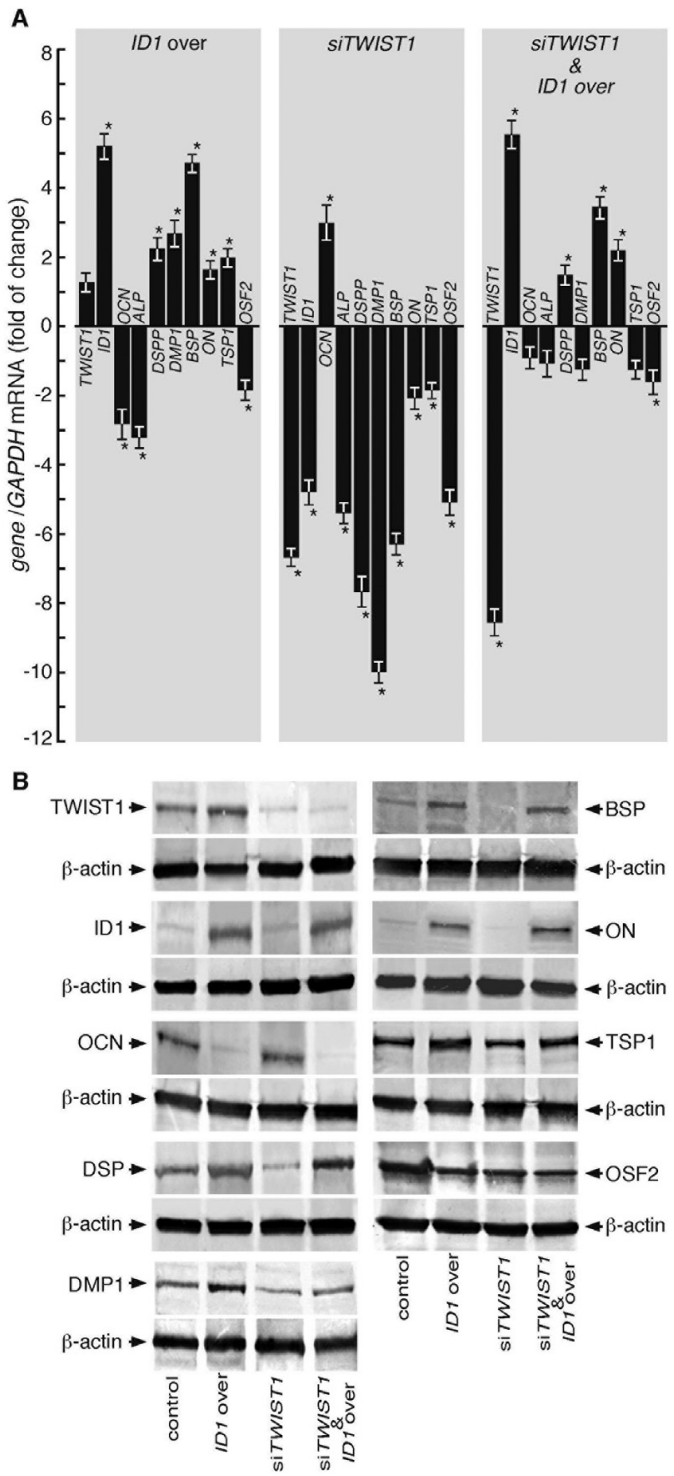

Figure 2. The effect of lentivirus-mediated alteration of TWIST1 and ID1 expression on markers of human DPSCs differentiation. (A) Quantitative RT-PCR analysis of TWIST1, ID1, OCN, ALP, DSPP, $D M P 1, B S P, O N, T S P 1$, and OSF2 expression in: DPSCs transduced with pWPI-GFP-ID1 (ID1over), DPSCs transduced with $3.7 \mathrm{pLL}$ vector carrying the TWIST1 siRNAs (siTWIST1), and DPSCs transduced with $3.7 \mathrm{pLL}$ vector carrying the TWIST1 siRNAs and subsequently with pWPI-GFP-ID1 vector (siTWIST1\&ID1over). Data are normalized to GAPDH mRNA level and are presented as fold-change relative to control DPSCs transduced with an empty vector or vector carrying scrambled sequence. Data present the mean values $\pm S D$ $(n=6) .{ }^{*} \mathrm{p}<0.05$ vs. control. (B) The TWIST1, ID1, OCN, DSP, DMP1, BSP, ON, TSP1, and OSF2 protein levels in: DPSCs with suppressed expression of TWIST1 (siTWIST1), DPSCs with overexpression of ID1 (ID1over), DPSCs with suppressed expression of TWIST1 and overexpression of ID1 (siTWIST1\&ID1over). The proteins in the cell extract were separated by SDS-PAGE and immunoblotted with appropriate antibodies. $\beta$-actin was used as a reference protein. The blots presented are representative of those obtained in 3 experiments performed on unique cell cultures.

DPSCs with silenced TWIST1 and concomitant overexpression of ID1 (Fig. 3C).

\section{DISCUSSION}

Our previous and current study showed that both genes modulate the dental pulp stem cells commitment 

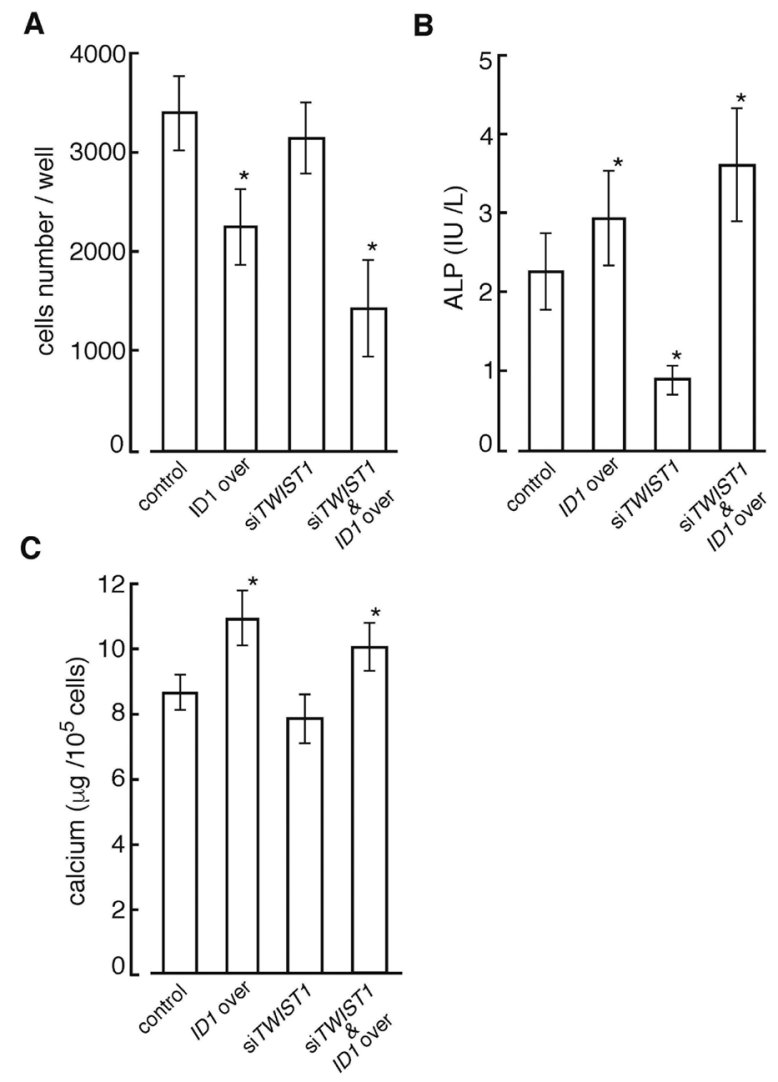

Figure 3. An impact of TWIST1 and ID1 expression level on DPSCs differentiation.

The cell proliferation (A), activity of alkaline phosphatase (ALP) (B), and calcium content (C) in cultures of: DPSCs with suppressed expression of TWIST1 (siTWIST1), DPSCs with overexpression of ID1 (ID1over), DPSCs with suppressed expression of TWIST1 and overexpression of ID1 (siTWIST1\&ID1over) were determined as described in Materials and Methods. The data are the mean values \pm S.D. $(n=3) .{ }^{*} p<0.05$ vs. control.

and differentiation and are responsible for pushing the DPSCs' to differentiation into odontoblast-like cells (Maciejewska et al., 2014). A similar conclusion from in vivo study has been just announced by Meng and coworkers (Meng et al., 2015). These authors showed that the Twist $2^{\text {cre }}+{ }^{+} ;$Twist $1^{\text {flf } f}$ mice embryos had smaller tooth germs with defective dentin and enamel compared to the controls and these defects reflected disturbances in odontoblasts differentiation. Our current in vitro study also showed that TWIST1 silencing repressed human DPSCs commitment, which appeared as the diminished expression of both the early and late markers of odontoblasts differentiation with the exception of osteocalcin. This observation was enhanced by the fact that DPSCs with silenced TWIST1 showed the diminished ALP activity and low concentration of calcium, which indicated the lack of their involvement in the mineralization process. The DPSCs with silenced TWIST1 showed morphologic features of undifferentiated cells with the high rate of proliferation compared to the controls. Thus, our results seem to confirm that TWIST1 is an essential factor for both the odontoblasts commitment and final differentiation and that in the DPSCs TWIST1 might act in the cell type specific manner, and it differs from that observed in osteoblasts (Bialek et al., 2004).

Multiple studies on TWIST1 role in bone development emphasized that the cessation of TWIST1 expression is the prerequisite for osteoblast differentiation (Bialek et al., 2004) but also suggested that osteoblasts and odontoblasts differentiation might be regulated by distinct mechanisms (Batouli et al., 2003). In bone it was shown that the outcome of TWIST1 expression depends on the balance of the concentration of TWIST1 homodimers and heterodimers, which is regulated by the co-expression of the ID1 protein. This dimerization mechanism was thoroughly investigated in the cranial sutures, palate and tooth (Connerney et al., 2006; Rice et al., 2005). Our previous study showed that the forced expression of ID1 in the DPSCs pushed the cells to differentiation into the odontoblast-like cells (Maciejewska et al., 2014). Thus, in the current study we intended to check whether the forced overexpression of ID1 might compensate the repressed differentiation of DPSCs caused by TWIST1 silencing. Indeed, the ID1 overexpression resulted in the increase of the DPSCs maturity regardless the dramatic decrease in TWIST1 expression. The modulated cells commenced the specific threshold of maturity, which appeared as the elevated expression of DSPP, DMP1, BSP, ON and TSP1 in comparison to that observed in the controls. The finding was enhanced by the increased activity of ALP and calcium concentration in the DPSCs (Twist $1^{-} / \mathrm{Id}^{+}$) compared to the DPSCs with silenced TWIST1. The observation of ID1-dependent increase in ALP activity was reported in osteoblast by Peng and coworkers who concluded that ID1 stimulated osteoblasts maturation by up-regulation of BMP9 expression (Peng et al., 2004). However, before the final osteoblasts maturation the ID1 expression must cease (Peng et al., 2004; Song et al., 2011). Rice and co-workers showed that the similar mechanisms operate in vivo (Rice et al., 2005). These previous reports clearly indicate that TWIST1 and ID1 must directly or indirectly interact in the cell type specific loop that regulates DPSCs development. So far the only confirmed explanation for the reported results is the prevailed formation of TWIST1 homodimers over heterodimers in the presence of ID1 (Connerney et al., 2006; Connerney et al., 2008). The dimerization data came from the experiment in M3T3-E1 cells, which showed that the forced expression of ID1 abrogated the TWIST1-dependent repression of BMP signaling (Hayashi et al., 2007). Since BMP signaling stimulates the expression of osteoblasts differentiation markers $\mathrm{Ju}$ et al., 2000) in C3H10T1/2 cells, it seems likely that in our experiment the same mechanism could be involved. This assumption is strengthened by the fact that in both our and others' experiments (Ju et al., 2000; Zhang et al., 2008) TWIST1 expression adversely modulated the expression of the osteocalcin gene. Thus, even if TWIST1 acts differently in the DPSCs and C3H10T1/2 cells the overexpression of ID1 might have overcome the results in the TWIST1 activity in the both cell lines.

Concluding, in our experiment we showed that the high level of ID1 expression might be a critical factor stimulating dental pulp stem cells to final differentiation and maturation. We also showed that the forced overexpression of ID1 might compensate the repressed differentiation of DPSCs caused by TWIST1 silencing. Since both proteins TWIST1 and ID1 are involved in the numerous signaling pathways, the further experiments should clarify the detailed direct or indirect connection between those two factors, which seems critical for the proper development of a tooth organ.

\section{Conflict of interest}

Authors deny any conflict of interest. 


\section{Acknowledgements}

Authors are grateful to Dr. Larry Fisher (National Institutes of Health/National Institute of Dental and Craniofacial Research) for providing anti-DSP and antiDMP1 antibodies.

Dr. Maciejewska directs her appreciation to Dr. Rena N. D'Souza and her team from Baylor College of Dentistry, Dallas, TX, for advice and education in the execution of these studies.

Support for education of Dr. Maciejewska and the studies described in this manuscript was provided by Grant R01 - DE 013368 NIDCR/NIH

This work was supported by grant from MNiSW NN 403128340 to IM.

\section{REFERENCES}

Batouli S, Miura M, Brahim J, Tsutsui TW, Fisher LW, Gronthos S et al. (2003) Comparison of stem-cell-mediated osteogenesis and dentinogenesis. J Dent Res 82: 976-981

Bialek P, Kern B, Yang X, Schrock M, Sosic D, Hong N et al. (2004) A twist code determines the onset of osteoblast differentiation. Dev Cell 6: 423-435. http://dx.doi.org/10.1016/S1534-5807(04)00058-9

Castanon I, Von SS, Kass J, Baylies MK (2001) Dimerization partners determine the activity of the Twist bHLH protein during Drosophila mesoderm development. Development 128: 3145-3159

Connerney J, Andreeva V, Leshem Y, Mercado MA, Dowell K, Yang $\mathrm{X}$ et al. (2008) Twist1 homodimers enhance FGF responsiveness of the cranial sutures and promote suture closure. Dev Biol 15: 323334. http://doi.org/10.1016/j.ydbio.2008.03.037

Connerney J, Andreeva V, Leshem Y, Muentener C, Mercado MA, Spicer DB (2006) Twist1 dimer selection regulates cranial suture patterning and fusion. Dev Dyn 235: 1345-1357. http://doi. org/10.1002/dvdy.20717

Gronthos S, Mankani M, Brahim J, Robey PG, Shi S (2000) Postnatal human dental pulp stem cells (DPSCs) in vitro and in vivo. Pro Natl Acad Sci U S A 97: 13625-13630. http://doi.org/10.1073/ pnas. 240309797

Hayashi M, Nimura K, Kashiwagi K, Harada T, Takaoka K, Kato H et al. (2007) Comparative roles of Twist-1 and Id1 in transcriptional regulation by BMP signaling. I Cell Sci 120: 1350-1357. http://doi. org $/ 10.1242 /$ jcs.000067

Ju W, Hoffmann A, Verschueren K, Tylzanowski P, Kaps C, Gross G, Huylebroeck D (2000) The bone morphogenetic protein 2 signaling mediator Smad1 participates predominantly in osteogenic and not in chondrogenic differentiation in mesenchymal progenitors C3H10T1/2. J Bone Miner Res 15: 1889-1899. http://doi. org/10.1359/jbmr.2000.15.10.1889

Laursen KB, Mielke E, Iannaccone P, Fuchtbauer EM (2007) Mechanism of transcriptional activation by the proto-oncogene Twist1.
I Biol Chem 282: 34623-34633. http://doi.org/10.1074/jbc. M707085200

Li Y, Lu Y, Maciejewska I, Galler KM, Cavender A, D’Souza RN (2011) TWIST1 promotes the odontoblast-like differentiation of dental stem cells. Adv Dent Res 23: 280-284. http://doi. org/10.1177/0022034511405387

Maciejewska I, Sakowicz-Burkiewicz M, Pawelczyk T (2014) Id1 expression level determines the differentiation of human dental pulp stem cells. J Dent Res 93: 576-581. http://doi.org/ 10.1177/0022034514530164

Meng T, Huang Y, Wang S, Zhang H, Dechow PC, Wang X et al. (2015) Twist1 is essential for tooth morphogenesis and odontoblast differentiation. J Biol Chem 290: 29593-29602. http://doi.org/ 10.1074/jbc.M115.680546.

Peng Y, Kang Q, Luo Q, Jiang W, Si W, Liu BA et al. (2004) Inhibitor of DNA binding/differentiation helix-loop-helix proteins mediate bone morphogenetic protein-induced osteoblast differentiation of mesenchymal stem cells. J Biol Chem 279: 32941-32949. http://doi. org/10.1074/jbc.M403344200

Rice DP, Aberg T, Chan Y, Tang Z, Kettunen PJ, Pakarinen L et al. (2000) Integration of FGF and TWIST in calvarial bone and suture development. Development 127: 1845-1855

Rice DP, Rice R, Thesleff I (2003) Molecular mechanisms in calvarial bone and suture development and their relation to craniosynostosis. Eur I Orthod 25: 139-148

Rice R, Thesleff I, Rice DP (2005) Regulation of Twist Snail and Id1 is conserved between the developing murine palate and tooth. Dev Dyn 234: 28-35. http://doi.org/10.1002/dvdy.20501

Sakowicz-Burkiewicz M, Kitowska A, Grden M, Maciejewska I, Szutowicz A, Pawelczyk T (2013) Differential effect of adenosine receptors on growth of human colon cancer HCT 116 and HT-29 cell lines. Arch Biochem Biophys 533: 47-54. http://doi.org/10.1016/j. abb.2013.02.007

Song X, Liu S, Qu X, Hu Y, Zhang X, Wang T, Wei F (2011) BMP2 and VEGF promote angiogenesis but retard terminal differentiation of osteoblasts in bone regeneration by up-regulating Id1. Acta Biochim Biophys Sin (Shanghai) 43: 796-804. http://doi.org/10.1093/ abbs/gmr074

Thisse C, Perrin-Schmitt F, Stoetzel C, Thisse B (1991) Sequencespecific transactivation of the Drosophila twist gene by the dorsal gene product. Cell 65: 1191-1201. http://dx.doi.org/10.1016/00928674(91)90014-P

Zhang Y, Blackwell EL, McKnight MT, Knutsen GR, Vu WT, Ruest LB (2012) Specific inactivation of Twist1 in the mandibular arch neural crest cells affects the development of the ramus and reveals interactions with hand2. Dev Dyn 241: 924-940. http://doi. org/10.1002/dvdy.23776

Zhang Y, Hassan MQ, Li ZY, Stein JL, Lian JB, van Wijnen AJ, Stein GS (2008) Intricate gene regulatory networks of helix-loop-helix $(\mathrm{HLH})$ proteins support regulation of bone-tissue related genes during osteoblast differentiation. J Cell Biochem 105: 487-496. http:// doi.org/ 10.1002/jcb.21844 